\title{
Dermatite verrucosa de Pedroso e Gomes (Cromomicose): 90 anos depois, ainda entre nós
}

\section{Pedroso and Gomes' Verrucous Dermatitis (Chromoblastomycosis): \\ 90 years on and still among us}

\author{
Paulo Ricardo Criado ${ }^{1}$ \\ Hebert Roberto Clivati Brandt ${ }^{3}$ \\ Ilana Halpern ${ }^{5}$
}

\author{
Neuza Yuriko Sakai Valente ${ }^{2}$ \\ Walter Belda Junior ${ }^{4}$
}

Resumo: Os autores relatam o caso clínico de uma doente que procurou atendimento dermatológico em decorrência da presença de lesão em placa papulosa de superfície verrucosa no cotovelo esquerdo. Palavras-chave: Cromoblastomicose; Dermatopatias; Micoses

\begin{abstract}
In this report, the authors describe the clinical case of a woman seeking care at this dermatology outpatient clinic with a verrucous plaque on her left elbow.

Keywords: Chromoblastomycosis; Mycoses; Skin diseases
\end{abstract}

Mulher de 75 anos, lavradora, proveniente da área rural de Ipatinga (MG), latitude - 19 $28^{\prime} 06^{\prime \prime}$, longitude $42^{\circ} 32^{\prime} 12^{\prime \prime}$, altitude de $240 \mathrm{~m}$. Referia lesão no cotovelo há cerca de dois anos. O exame anatomopatológico estabeleceu o diagnóstico de cromomi$\operatorname{cose}$, a qual foi descrita em periódico brasileiro há 90
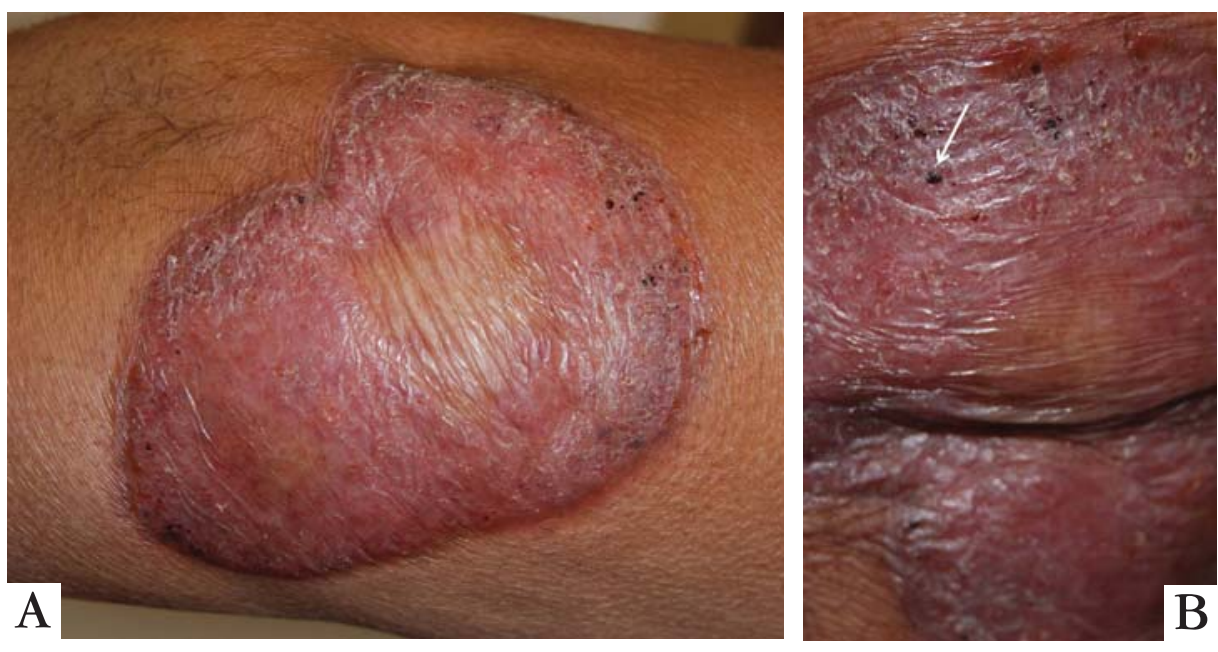

Figura 1: A. Lesão eritematoescamosa crostosa no cotovelo esquerdo da doente;

B. Detalhe da lesão; a seta indica crosta hemática onde, preferencialmente, o material deve ser colhido para exame micológico, obtendo-se o achado mais frequente dos corpos fumagoides

Aprovado pelo Conselho Editorial e aceito para publicação em 23.12.2009

* Trabalho realizado na Divisão de Dermatologia do Hospital das Clínicas da Faculdade de Medicina da Universidade de São Paulo (HCFMUSP) - São Paulo (SP), Brasil. Conflito de interesse: Nenhum / Conflict of interest: None

Suporte financeiro: Nenhum / Financial funding: None

Doutor em Ciências (Dermatologia) pela Faculdade de Medicina da Universidade de São Paulo (FMUSP), médico da Divisão de Dermatologia e investigador do LIM-53 (Micologia Médica) do Hospital das Clínicas da Faculdade de Medicina da Universidade de São Paulo (HCFMUSP) - São Paulo (SP), Brasil.

Doutora em Ciências (Dermatologia), dermatopatologista da Divisão de Dermatologia do Hospital das Clínicas da Faculdade de Medicina da Universidade de São Paulo (HCFMUSP), investigadora do LIM-53 (Micologia Médica) do Hospital das Clínicas da Faculdade de Medicina da Universidade de São Paulo (HCFMUSP) - São Paulo (SP), Brasil.

Dermatologista pela Sociedade Brasileira de Dermatologia (SBD), dermatologista do Serviço de Dermatologia do Hospital do Servidor Público Municipal de São Paulo - São Paulo (SP), Brasil.

Livre-docente pela Universidade Estadual de Campinas (Unicamp), doutor pela Faculdade de Medicina da Universidade de São Paulo (USP), professor de Dermatologia da Faculdade de Medicina da Universidade de São Paulo (USP) - São Paulo (SP), Brasil.

Patologista do Setor de Dermatopatologia da Divisão de Dermatologia do Hospital das Clínicas da Faculdade de Medicina da Universidade de São Paulo (HCFMUSP) - São Paulo (SP), Brasil. 


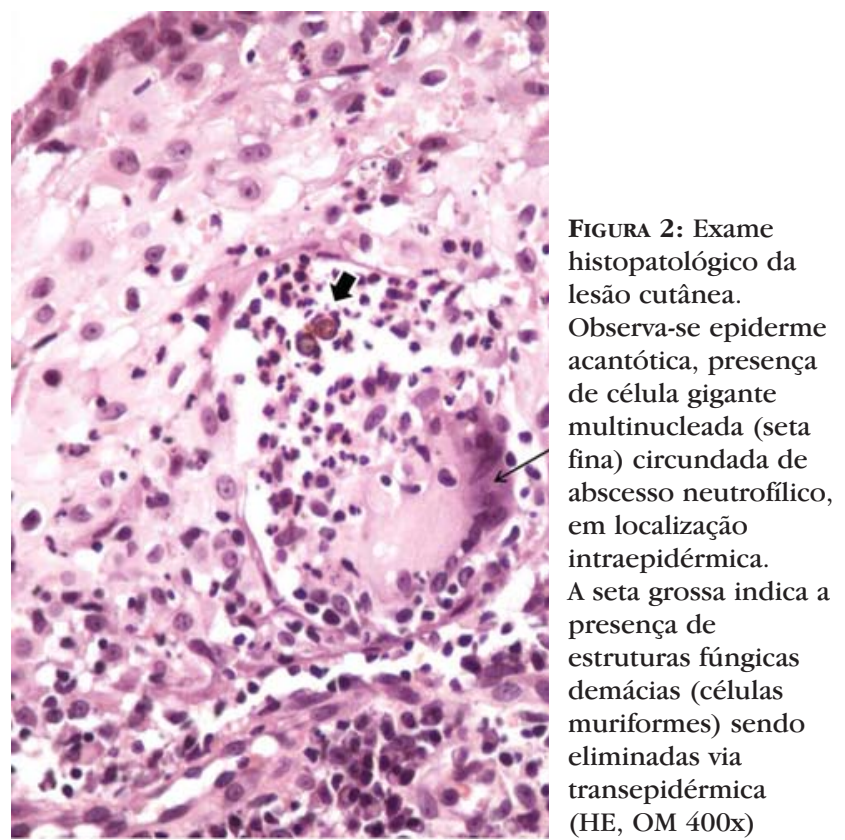

\section{REFERÊNCIAS}

1. Pedroso A, Gomes JM. Sobre quatro casos de dermatite verrucosa produzida por Phialophora verrucosa. An Paul Med Cir. 1920;11:53-61.

2. Rudolph M. ber die brasilianische 'Figueira' (Vorläufige Mitteilung). Archiev Schiffs und Tropen-Hyg 1914;18:498-9.

3. Silva CMP, Marques SG, Silva RB, Sousa Jr SS, Menezes Jr DRT, Costa JML. Cromoblastomicose tratada com itraconazol sistêmico associado a anfotericina B intralesional. An Bras Dermatol. 1999;74:41-4. anos por Alexandrino Pedroso e José Maria Gomes. ${ }^{1}$ A primazia da descrição deve-se ao médico alemão Max W. Rudolph (1887-1955), que estudou seis casos da doença conhecida como "figueira" em 1914. ${ }^{2}$ A cromomicose constitui uma infecção fúngica crônica da epiderme, derme e tecido celular subcutâneo, que ocorre em climas tropicais, bem como em regiões temperadas da América Latina, onde o agente etiológico mais comum é a Fonsecaea pedrosoi, ${ }^{3}$ fungo que foi isolado a partir da coleta de fragmento de pele da paciente em questão.

\author{
ENDEREÇO PARA CORRESPONDÊNCIA / MAILING ADDRESS: \\ Paulo Ricardo Criado \\ Rua Carneiro Leão, 33 \\ Vila Scarpelli \\ 09050430 Santo André, SP
}

Como citar este artigo/How to cite this article: Criado PR, Valente NYS, Brandt HRC, Belda Jr W, Halpern I. Dermatite verrucosa de Pedroso e Gomes (Cromomicose): 90 anos depois, ainda entre nós. An Bras Dermatol. 2010;85(1):104-5. 